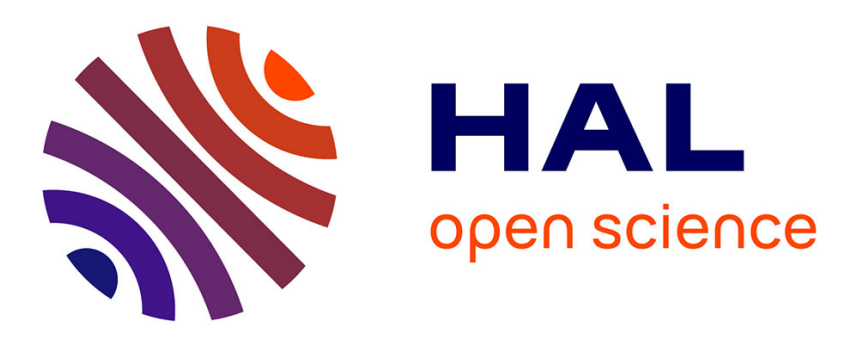

\title{
Analyse cytogénétique et comportement vis-à-vis de Septoria nodorum d'hybrides Triticum sp. x Aegilops squarrosa et de leurs descendances
}

\author{
Maxime Trottet, Françoise Dosba, Anne-Marie Tanguy
}

\section{To cite this version:}

Maxime Trottet, Françoise Dosba, Anne-Marie Tanguy. Analyse cytogénétique et comportement visà-vis de Septoria nodorum d'hybrides Triticum sp. x Aegilops squarrosa et de leurs descendances. Agronomie, 1983, 3 (7), pp.659-664. hal-00884556

\section{HAL Id: hal-00884556 https://hal.science/hal-00884556}

Submitted on 1 Jan 1983

HAL is a multi-disciplinary open access archive for the deposit and dissemination of scientific research documents, whether they are published or not. The documents may come from teaching and research institutions in France or abroad, or from public or private research centers.
L'archive ouverte pluridisciplinaire $\mathbf{H A L}$, est destinée au dépôt et à la diffusion de documents scientifiques de niveau recherche, publiés ou non, émanant des établissements d'enseignement et de recherche français ou étrangers, des laboratoires publics ou privés. 


\title{
Analyse cytogénétique et comportement vis-à-vis de Septoria nodorum d'hybrides Triticum sp. $\times$ Aegilops squarrosa et de leurs descendances
}

\author{
Maxime TROTTET \& Françoise DOSBA (1) \\ avec la collaboration technique de Annc-Maric TANGUY \\ I.N.R.A., Station d'Amélioration des Plantes, Centre de Recherches de Rennes, B.P. 29 F 35650 Le Rheu
}

RÉSUMÉ

\begin{abstract}
Des croisements ont été réalisés entre Aegilops squarrosa $\mathrm{n}^{\circ} 33$ résistant à Septoria nodorum et divers blés. Le comportement méiotique très irrégulier en F1 pour certaines lignées (pourcentage élevé de multivalents) s'améliore fortement en F2. Le comportement vis-à-vis de la septoriose présente une grande variabilité et laisse entrevoir la possibilité de sélectionner des lignées plus courtes et plus résistantes que "Comtal » à partir de ce matéricl.
\end{abstract}

Mots clés additionnels : Analyse cytologique, résistance aux parasites. and Aegilops squarrosa and of their progenies.

Crosses have been made between Aegilops squarrosa n 33 resistant to Septoria nodorum and various wheats. Meiotic behaviour was very irregular in some of the F1 lines duc to a high percentage of multivalents but improved greatly in the F2. Glume blotch reaction showed considerable variability, so that it may be possible to breed from this material lines shorter and more resistant than "Comtal".

Additional key words : Cytogenetic analysis, disease resistance.

\section{INTRODUCTION}

La septoriose provoquée par Septoria nodorum Berk. est une maladie grave du blé dans l'Europe du Nord-Ouest. En France, cette maladie était la plus fréquente en 1980 et une des principales causes de diminution des rendements en 1981. L'ensemble des variétés cultivées est sensible à la septoriose. Seules quelques variétés anciennes ou exotiques de faible valeur agronomique et de taille élevée présentent un bon niveau de résistance vis-à-vis de ce parasite. La liaison résistance-taille élevée semble difficile à rompre soit par sélection de transgressions après hybridation (BRÖNNIMANN, 1974), soit par mutagenèse (FossATI \& BRÖNNIMANN, 1975). La prospection au sein des espèces voisines du blé tendre a permis de mettre en évidence le bon comportement de certains Aegilops squarrosa L., en particulier de la lignée ${ }^{\circ} 33$ (JAHIER \& TROTTET, 1980).

(1) Nouvelle adresse : I.N.R.A., Station d'Arboriculture fruitière, Centre de Recherches de Bordeaux, F 33140 Pont-de-laMaye.
Des hybridations entre cette lignée et différents blés ont été réalisées dans le but de créer des géniteurs blé ayant un haut niveau de résistance à la septoriose et une taille analogue à celle des blés cultivés. Cet article présente quelques caractéristiques de descendances de croisements entre Ae. squarrosa $\mathrm{n}^{\circ} 33$ et des blés tendres.

\section{MATÉRIEL ET MÉTHODES}

\section{A. Le matériel végétal}

En 1975, nous avons réalisé divers croisements entre $A e$. squarrosa $\mathrm{n}^{\circ} 33$ choisi comme parent femelle ou parent mâle et des blés tétraploïdes ( $T$. dicoccum, $T$. durum, $T$. dicoccoìdes, $T$. persicum) ou des blés tendres.

Les 2 seuls croisements ayant donné des plantes F1 viables sont :

- Ae. squarrosa $\times T$. dicoccum 119. 
L'hybride Ae. squarrosa $\times T$. durum n'a donné de descendance ni en autofécondation ni en croisement et les traitements à la colchicine pour doubler le nombre de chromosomes ont échoué.

Seul le $2^{c}$ croisement a donné une descendance en fécondation libre et en croisement avec une lignéc de blé de printemps ("Champlein» $x$ "Aronde ») 68 (fig. 1). Les 2 plantes viables constituant cette descendance ont été conduites en fécondation libre (FL) et en croisement avec une lignée sœur de "Roazon " ( VPM » $x$ « Moisson ») 41-2-3-3-11 (VM 4): une seule des 2 plantes était autofertile ct a pu être conduite en autofécondation.

\section{B. Méthodes cytologiques}

Le dénombrement chromosomique est réalisé à partir des méristèmes racinaires issus de grains mis à germer en boite de Pétri. Les 3 racines séminales sont prélevées et prétraitées dans l' $\alpha$ bromonaphtalène pendant $16 \mathrm{~h}$ à $4{ }^{\circ} \mathrm{C}$. Elles sont ensuite fixées dans l'acide acétique à 90 p. 100 pendant $39 \mathrm{mn}$, rincées puis stockées dans l'alcool ou colorées directement en utilisant la réaction nucléale de Feulgen après hydrolyse dans $\mathrm{HCL}(1 \mathrm{~N}), 12 \mathrm{mn}$ à $60^{\circ} \mathrm{C}$. L'obscrvation des méioses est réalisée à partir de cellules mères de grains de pollen en métaphase I dans du carmin acétique ferrique après fixation des étamines dans le Carnoy additionné de perchlorure de fer et stockage dans l'alcool à $70{ }^{\circ} \mathrm{C}$ (Dosba \& Doussinault, 1978).

\section{Méthodes de mesure du comportement vis-à-vis de $S$. nodorum}

Le dispositif expérimental était un criss-cross dans lequel chaque bloc était subdivisé en 2 sous-blocs séparés par une bande d'1 m de largeur de la variété "Huntsman». Un des sous-blocs était contaminć par $S$. nodorum, l'autre servait de témoin. La parcelle élémentaire était composéc de 2 lignes de 1,5 $\mathrm{m}$ de longueur séparées par 2 lignes de la variété «Huntsman". Etant donné le faible nombre de grains dont nous disposions, nous n'avons pu faire qu'une répétition en 1979 et 2 répétitions en 1980. Pour éviter la verse, un filet à mailles $15 \times 15 \mathrm{~cm}$ était tendu sur chaque sous-bloc à $40 \mathrm{~cm}$ du sol. Ce dispositif a été très efficace en 1979 mais a été insuffisant pour empêcher la verse d'unc partie de l'essai en 1980 du fait des tempêtes du mois de juin.

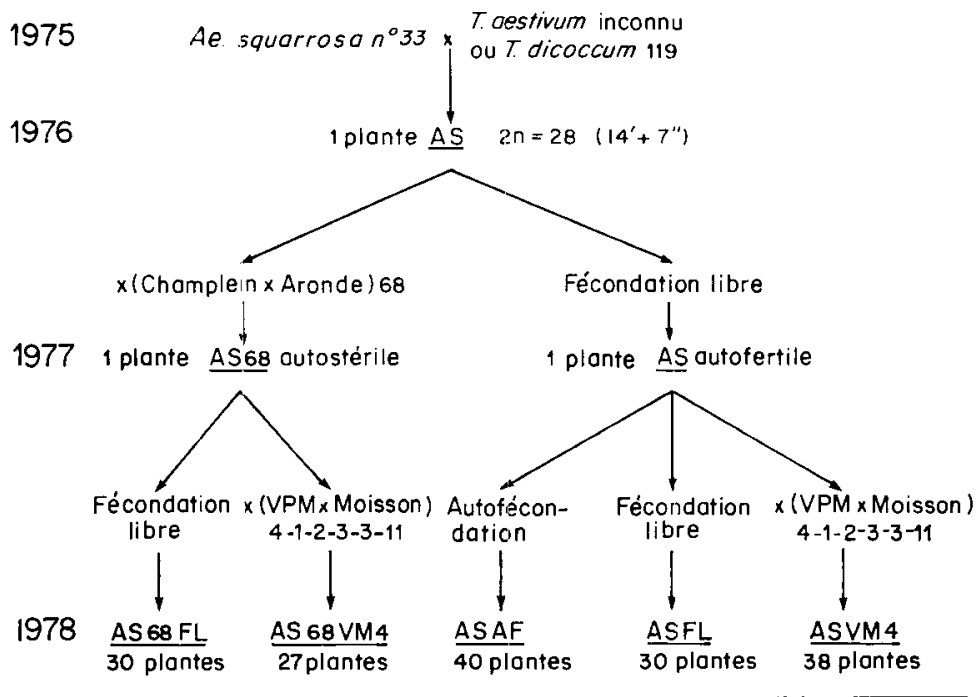

Plontes autofertiles et outofécondées récolte des grains sur les épis ensachés
Les lignes à tester étaient comparées à un blé tendre sensible, "Capitole", et au plus résistant à $S$. nodorum des blés tendres cultivés en France, "Comtal».

La contamination a été réalisée par pulvérisation d'unc suspension de spores à la concentration de $10^{6}$ spores $/ \mathrm{ml}$, jusqu'au début du ruissellement soit environ $100 \mathrm{ml} / \mathrm{m}^{2}$ de suspension. La contamination a cu lieu à l'épiaison de chaque lignéc et a ćté répétée 2 fois à 2 ou $3 \mathrm{j}$ d'intervalle.

Un dispositif d'irrigation par aspersion permettait de favoriser le développement de la maladic: la durée de chaque irrigation était de $20 \mathrm{mn}$ et le débit d'eau de $6 \mathrm{~mm} / \mathrm{h}$. L'irrigation était réalisée le jour de l'inoculation et ensuite tous les $2 \mathrm{j}$, lorsqu'il ne pleuvait pas, jusqu'à la fin du mois de juin.

A maturité, nous avons mesuré la taille de 15 plantes par parcelle ćlémentaire pour estimer la taille moyenne de chaque famille et récolté 50 ćpis par parcelle élémentairc. Ces épis ont été stockés $15 \mathrm{j}$ dans un grenicr à une température voisine de $35^{\circ} \mathrm{C}$ pour les amener à un taux d'humidité identique et faible. Ces épis ont ensuite été battus en mélange et nous avons estimé le poids de 1000 grains de chaque parcelle par comptage et pesée de 500 grains. Nous avons exprimé la valeur du poids de 1000 grains dans la parcelle inoculćc en fonction de celle de la parcelle témoin et nous appelons ce pourcentage, poids relatif de 1000 grains. Cette valeur donne la meilleure estimation du comportement vis-à-vis de $S$. nodorum (TRotTet \& MERIEN, 1982).

\section{RÉSULTATS}

\section{A. Analyse cytologique du matériel}

Les hybrides interspécifiques analysés à la génération F1 présentent un comportement méiotique régulier.

L'hybride Ae. squarrosa $\mathrm{n}^{\circ} 33 \times T$. durum cv. CF 84 présente en moyenne 20,88 univalents et 0,06 bivalents sur 70 cellules analysées.

Le traitement à la colchicine réalisé n'a pas permis de doubler le nombre chromosomique et la seulc plante obtenue, totalement stérile, n'a donné aucune descendance.

L'hybride Ae. squarrosa $\mathrm{n}^{\circ} 33 \times T$. dicoccum $\mathrm{n}^{\circ} 119$, analysé à partir de 2 plantes ct 160 cellules, préscnte 28 chromosomes au lieu des 21 attendus, 14,08 univalents et 6,96 bivalents, en moycnnc, en métaphase I.
Figure 1

Schéma d'obtention des lignées $A S$. Ways of obtaining AS lines. 
2 explications peuvent être envisagées pour expliquer le nombre chromosomique observé :

- c'est un gamète non réduit à $2 n=14$ chromosomes d'Ae. squarrosa qui a été pollinisé par un gamète de T. dicoccum;

- c'est un gamc̀te de $T$. aestivum à 21 chromosomes qui a pollinisć accidentellement Ae. squarrosa.

Le fait que la méiose de la plante hybride à $2 \mathrm{n}=28$ chromosomes ne présente pas de multivalents pourrait être en faveur de l'hypothèse d'un gamète non réduit d'Ae. squarrosa, mais il n'exclut pas l'autre hypothèse: il s'agirait alors d'un blé tendre ayant un génome $\mathrm{D}$ de même structure chromosomique qu'Ae. squarrosa $\mathrm{n}^{\circ} 33$.

L'étude de l'évolution cytologique du matériel fait apparaître des cas fréquents d'aneuploïdie après croisement par la lignćc « Champlein $\times$ Aronde $68 »-$ AS68 - (tabl. 1). Des plantes ayant jusqu'à 49 chromosomes ont pu être observées en 1978. Ce phénomène d'aneuploïdie se retrouve aussi chez l'hybride «AS68 VM4 », alors qu'il est moins important chez «ASVM4 ». Le nombre chromosomique des plantes AS et AS68 n'a pas été déterminé, on peut cependant supposer compte tenu du nombre chromosomique de leur descendance que AS avait 42 chromosomes tandis que AS68 en présentait un nombre plus important ce

\section{TABLEAU 1}

Nombre de chromosomes des plantes observées en 1978 et 1979. Chromosome number of the plants observed in 1978 and 1979.

\begin{tabular}{lllcc}
\hline \hline \multirow{2}{*}{ Année } & \multirow{2}{*}{ Généalogie } & \multicolumn{3}{c}{ Nombre de plantes avec } \\
\cline { 3 - 5 } & & $2 \mathrm{n}<42$ & $2 \mathrm{n}=42$ & $2 \mathrm{n}>42$ \\
\hline & & & & 7 \\
1978 & AS68 FL & & 3 & 1 \\
1979 & AS68 FL & 1 & 2 & 29 \\
1978 & AS68 VM4 & 1 & 6 & 14 \\
1979 & AS68 VM4 & 1 & 12 & \\
1978 & ASAF & 1 & 11 & \\
1979 & ASAF & 1 & 8 & 1 \\
1978 & ASFL & 12 & 50 & 2 \\
1978 & ASVM4 & 12 & \\
1979 & ASVM4 & & & \\
\hline \hline
\end{tabular}

qui pourrait expliquer son autostérilité. Le nombre chromosomique élevé d'AS68 pourrait provenir d'une méiose asyndétique de l'amphiploïde dans laquelle tous ou presque tous les chromosomes auraient migré au même pôle.

L'analyse du comportement méiotique des différentes origines ou «lignées » en 1978 et 1979 traduit de grandes irrégularités lorsque "Champlein $\times$ Aronde 68 » et (ou) "VM4 " sont impliqués dans les croisements de départ (tabl. 2). Le pourcentage de chromosomes sous forme de multivalents, très élevé en 1978, baisse généralement en 1979, sauf pour le croisement «AS68 VM4 » analysé au niveau d'une seule plante cette année-là. Les anomalies méiotiques qui s'atténuent au cours du temps sont vraisemblablement dues à des différences de structure chromosomique entre les blés mis en présence. Il a été démontré que «V.P.M. », « Moisson » et « Roazon», sélectionné à partir du croisement VPM $\times$ «Moisson», différent du blé « Chinese Spring » (blé de référence pour l'étude des translocations) par au moins 1 ou 2 translocations (BOURGEOIS et al., 1978). Si l'on compare le comportement de «VM4 " à celui de "Roazon », en croisement avec «Chinese Spring », on observe un fort pourcentage de cellules présentant 2 trivalents ou quadrivalents analogue à ce que l'on observe chez l'hybride "Moisson" $x$ "Chinese Spring ». BOURGEOIS et al. (1978) ont montré que «VPM» et « Roazon» différaient de «Chinese Spring» par la translocation 5B-7B tandis que «Moisson » différait par 2 translocations 5B-7B et $1 \mathrm{~B}-6 \mathrm{~B}$. Il est vraisemblable que «VM4 » diffère aussi de «Chinese Spring" par ces 2 mêmes translocations (tabl. 3). Par ailleurs, si l'on compare le comportement de «VPM » et de «(Champlein $\times$ Aronde) 68 » à une même lignée demi-naine d'origine mexicaine «Naïnari $60 \mathrm{~S}$ ", on peut penser que «(Champlein $\times$ Aronde) 68 " diffère de cette lignée par une translocation réciproque, tandis que VPM en diffère par 2 translocations.

En définitive, les différences de structure entre les blés mis en présence lors des différents croisements ainsi que le comportement très régulier de la famille ASAF dès 1978 nous amènent à conclure que les anomalies méiotiques observées sont en majeure partie dues à la présence de translocations réciproques. Comme l'ont déjà montré DosBA \& DOUSSinAult (1978) et comme le laisse supposer le tableau 2, elles s'atténueront au cours des générations.

TABLEAU 2

Evolution méiotique du matériel issu de l'hybride Ae. squarrosa $n^{\circ} 33 \times$ blé tendre. Change in meiotic behaviour of progeny of the hybrid Ae. squarrosa $n^{\circ} 33 \times$ bread wheat.

\begin{tabular}{|c|c|c|c|c|c|c|c|c|c|}
\hline \multirow{2}{*}{ Année } & \multirow{2}{*}{ Généalogie } & \multirow{2}{*}{$\begin{array}{l}\text { Nombre de } \\
\text { plantes }\end{array}$} & \multirow{2}{*}{$\begin{array}{l}\text { Nombre de } \\
\text { cellules }\end{array}$} & \multirow{2}{*}{$2 n$} & \multicolumn{5}{|c|}{$\%$ de chromosomes sous forme de } \\
\hline & & & & & I & II & III & IV & Autres \\
\hline 1978 & AS68 FL & 1 & 15 & 47 & 6,96 & 72,34 & 19,57 & 1,13 & \\
\hline 1979 & AS68 FL & 1 & 50 & 43 & 1,53 & 92,74 & 4,19 & 0,37 & 1,16 \\
\hline 1978 & AS68 VM4 & 3 & 77 & 41 à 46 & 6,99 & 76,19 & 9,74 & 7,08 & \\
\hline 1979 & AS68 VM4 & 1 & 11 & $43+t$ & 6,00 & 74,38 & 6,82 & 10,73 & 2,07 \\
\hline 1978 & ASAF & 1 & 27 & 42 & 1,59 & 98,41 & & & \\
\hline 1979 & ASAF & 1 & 30 & 42 & 0,64 & 98,41 & & 0,95 & \\
\hline 1978 & ASVM4 & 5 & 220 & 42 & 2,67 & 82,10 & 2,34 & 12,77 & 0,12 \\
\hline 1979 & ASVM4 & 12 & 540 & 41 à 44 & 1,01 & 93,58 & 0,69 & 4,61 & 0,11 \\
\hline
\end{tabular}




\section{TABLEAU 3}

Pourcentage de cellules présentant un ou plusieurs multivalents chez des hybrides F1 entre différents blés. Percentage of cells with one or several multivalents in F1 hybrids between different wheat lines.

\begin{tabular}{|c|c|c|c|c|c|c|}
\hline \multirow[b]{2}{*}{ Croisements } & \multirow{2}{*}{$\begin{array}{l}\text { Nombre } \\
\text { de } \\
\text { plantes }\end{array}$} & \multirow{2}{*}{$\begin{array}{l}\text { Nombre de } \\
\text { cellules } \\
\text { observées }\end{array}$} & \multicolumn{4}{|c|}{ Pourcentage de cellules avec } \\
\hline & & & $\begin{array}{l}1 \text { III ou } \\
1 \text { IV }\end{array}$ & $\begin{array}{l}2 \text { III ou IV } \\
\text { ou } 1 \text { autre } \\
\text { multivalent }\end{array}$ & $\begin{array}{l}3 \text { III ou IV } \\
\text { ou } 2 \text { autres } \\
\text { multivalents }\end{array}$ & $\begin{array}{l}4 \text { III ou IV } \\
\text { (2 III ou IV) } \\
\text { + multi.) }\end{array}$ \\
\hline \multirow{6}{*}{ 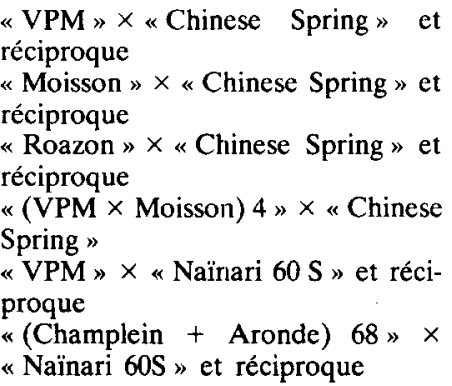 } & 19 & 615 & 81,14 & 9,92 & 1,62 & \\
\hline & 20 & 644 & 59,63 & 33,08 & 2,79 & \\
\hline & 8 & 360 & 79,44 & 6,94 & 0 & \\
\hline & 6 & 299 & 36,45 & 54,18 & 6,69 & \\
\hline & 14 & 554 & 59,57 & 30,80 & 1,81 & 0,36 \\
\hline & 11 & 439 & 39,64 & 4,50 & 0,45 & \\
\hline $\begin{array}{l}\text { III = trivalent. } \\
\text { IV = quadrivalent. }\end{array}$ & & & & & & \\
\hline
\end{tabular}

\section{B. Comportement du matériel vis-à-vis de $S$. nodorum}

Nous avons commencé à étudier le comportcment vis-àvis de $S$. nodorum en 1979 sur des F2 pour les familles ASVM4, AS68 VM4 et AS68 FL, sur des F3 pour ASAF ct sur un mélange de $\mathrm{F} 2$ et $\mathrm{F} 3$ pour ASFL.

En 1979, l'attaque après contamination artificielle par $S$. nodorum a été d'importance moyenne: le poids de 1000 grains du témoin sensible ( Capitole ») a été fortement diminué alors que celui du témoin modérément résistant («Comtal») a été peu touché (tabl. 4). Toutefois les essais de comportement vis-à-vis de $S$. nodorum étaient précis. Pour des essais voisins avec 2 ou 4 répétitions, le coefficient de variation était toujours compris entre 4 et 6 p. 100 . En 1980 , de fortes pluies ont permis un dévcloppcment très important de $S$. nodorum après contamination artificielle, mais la septoriose a été peu importante dans les parcelles témoins non contaminées. Dans ces conditions la diminution de poids de 1000 grains de «Comtal» est forte (tabl. 4). Toutefois le coefficient moyen de variation de l'essai était de 13,6 p. $100 \mathrm{du}$ fait d'une verse importante provoquée par les pluies.

En 1979 comme en 1980, (tabl. 4), nous constatons que le comportement moyen des lignées (mesuré par le poids relatif de 1000 grains) est proche de celui de «Comtal». Les lignées qui n'ont pas été recroisées (ASAF et ASFL) ont, en moyenne, un comportement supérieur à celui des lignées qui ont été recroisées. Mais la variation entre les lignées de chaque famille est importante sauf pour AS68FL en 1978 , où le nombre de lignées était trop faible et, dans toutes les familles, nous trouvons en 1979 et en 1980 des lignées ayant ur meilleur comportement que «Comtal» alors que d'autres sont plus sensibles que «Capitole".

La taille des pllantes (tabl. 5) cst en moyenne ćlcvée bien qu'il y ait une assez grande variabilité pour ce caractère. En 1979, les familles les plus courtes avaient la taille de «Comtal». En 1980, quelques familles sont aussi courtes que « Capitole». Les plantes les plus courtes ont tendance à être plus sensibles à la septoriose, le coefficient de corrélation entre la taille et le poids de 1000 grains relatif ćtait $r=0,49^{*}$ en 1979 et $r=0,38^{*}$ en 1980 . Remarquons que, dans tous nos essais, en 1980, la liaison entre la taille et le comportement vis-à-vis de $S$. nodorum a été beaucoup plus faible qu'en 1979, du fait d'une verse importante en 1980. Cependant, bien que les familles les plus courtes aient tendance à être plus sensibles à la septoriose, la différence de sensibilité est faible et l'on trouve dans ce matéricl des plantes plus courtes que "Comtal" avec un meilleur comportement vis-à-vis de $S$. nodorum.

\section{Comportement vis-à-vis des autres parasites}

Bien qu'Ae. squarrosa $\mathrm{n}^{\circ} 33$ soit résistant à l'oïdium et peu sensible aux rouilles jaune et brune, presque toutes les

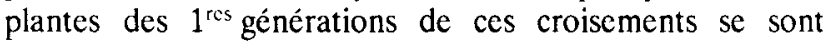
montrées très sensibles à ces 3 maladies. En 1980, aucune famille n'avait un bon comportement vis-à-vis de l'oïdium bien qu'une certaine variabilité pour ce caractère ait été observée. Pour les rouilles, la variabilité du comportement est plus importante, mais les plantes résistantes aux rouilles jaune et/ou brune se trouvent surtout dans les familles issues des croisements avec "VM4" et/ou "(Champlein $\times$ Aronde) $68 »$.

\section{DISCUSSION ET CONCLUSION}

L'utilisation d'Ae. squarrosa a permis de créer un matériel original et d'introduire chez le blé tendre une source nouvelle de résistance à $S$. nodorum. Certaines lignées présentent un niveau élevé de résistance ce qui montre qu'une partic au moins de la résistance à $S$. nodorum d'Ae. squarrosa pcut s'exprimer dans le génotype du blé tendre.

Du point de vue cytologique, en dépit de l'aneuplö̈die et des irrégularités méiotiques observées dans les $1^{\text {res }}$ générations et entraînant un faible niveau de fertilité des plantes, il ne semble pas y avoir de problèmes majeurs pour l'exploitation du matériel obtenu. L'instabilité du matériel dans les $1^{\text {res }}$ générations implique seulement de réaliser une sélection peu intense au départ. 
TABLEAU 4

Comportement des lignées vis-à-vis de S. nodorum en 1979 et 1980 (poids relatif de mille grains). Behaviour of the lines towards S. nodorum in 1979 and 1980 (relative 1000-grain weight).

\begin{tabular}{|c|c|c|c|c|c|}
\hline Familles & & $\begin{array}{l}\text { Nombre de } \\
\text { lignées }\end{array}$ & Moyenne & Ecart-type & Extrêmes \\
\hline $\begin{array}{l}\text { ASAF } \\
\text { ASAF }\end{array}$ & $\begin{array}{l}1979 \\
1980\end{array}$ & $\begin{array}{r}8 \\
14\end{array}$ & $\begin{array}{l}96,01 \\
78,21\end{array}$ & $\begin{array}{r}2,22 \\
10,33\end{array}$ & $\begin{array}{r}100,39-93,08 \\
99,10-61,64\end{array}$ \\
\hline $\begin{array}{l}\text { ASFL } \\
\text { ASFL }\end{array}$ & $\begin{array}{l}1979 \\
1980\end{array}$ & $\begin{array}{l}12 \\
15\end{array}$ & $\begin{array}{l}96,00 \\
79,23\end{array}$ & $\begin{array}{l}5,60 \\
7,67\end{array}$ & $\begin{array}{r}103,85-86,30 \\
91,43-67,50\end{array}$ \\
\hline $\begin{array}{l}\text { ASVM4 } \\
\text { ASVM4 }\end{array}$ & $\begin{array}{l}1979 \\
1980\end{array}$ & $\begin{array}{l}23 \\
32\end{array}$ & $\begin{array}{l}93,72 \\
73,13\end{array}$ & $\begin{array}{l}6,66 \\
7,77\end{array}$ & $\begin{array}{r}105,91-81,49 \\
95,69-61,45\end{array}$ \\
\hline $\begin{array}{l}\text { AS68 FL } \\
\text { AS68 FL }\end{array}$ & $\begin{array}{l}1979 \\
1980\end{array}$ & $\begin{array}{r}2 \\
11\end{array}$ & $\begin{array}{l}91,74 \\
76,01\end{array}$ & $\begin{array}{l}0,59 \\
9,35\end{array}$ & $\begin{array}{l}92,16-91,32 \\
87,70-61,86\end{array}$ \\
\hline $\begin{array}{l}\text { AS68 VM4 } \\
\text { AS68 VM4 }\end{array}$ & $\begin{array}{l}1979 \\
1980\end{array}$ & $\begin{array}{r}4 \\
13\end{array}$ & $\begin{array}{l}89,60 \\
73,40\end{array}$ & $\begin{array}{l}7,07 \\
9,74\end{array}$ & $\begin{array}{l}95,74-79,66 \\
87,75-56,38\end{array}$ \\
\hline $\begin{array}{l}\text { Moyenne des familles } \\
\text { Moyenne des familles }\end{array}$ & $\begin{array}{l}1979 \\
1980\end{array}$ & $\begin{array}{l}49 \\
85\end{array}$ & $\begin{array}{l}94,29 \\
76,96\end{array}$ & & \\
\hline $\begin{array}{l}\text { Capitole } \\
\text { Capitole }\end{array}$ & $\begin{array}{l}1979 \\
1980\end{array}$ & & $\begin{array}{l}66,99 \\
64,28\end{array}$ & & \\
\hline $\begin{array}{l}\text { Comtal } \\
\text { Comtal }\end{array}$ & $\begin{array}{l}1979 \\
1980\end{array}$ & & $\begin{array}{l}96,85 \\
75,53\end{array}$ & & \\
\hline
\end{tabular}

TABLEAU 5

Hauteur des familles en 1979 et 1980.

Plant height of the families in 1979 and 1980.

\begin{tabular}{|c|c|c|c|c|c|}
\hline Familles & & $\begin{array}{l}\text { Nombre de } \\
\text { lignées }\end{array}$ & Moyenne & Ecart-type & Extrêmes \\
\hline $\begin{array}{l}\text { ASAF } \\
\text { ASAF }\end{array}$ & $\begin{array}{l}1979 \\
1980\end{array}$ & $\begin{array}{r}8 \\
14\end{array}$ & $\begin{array}{l}149,63 \\
129,57\end{array}$ & $\begin{array}{r}5,34 \\
15,41\end{array}$ & $\begin{array}{rr}158 & -146 \\
145 & -85\end{array}$ \\
\hline $\begin{array}{l}\text { ASFL } \\
\text { ASFL }\end{array}$ & $\begin{array}{l}1979 \\
1980\end{array}$ & $\begin{array}{l}12 \\
15\end{array}$ & $\begin{array}{l}138,90 \\
132,50\end{array}$ & $\begin{array}{l}10,75 \\
12,09\end{array}$ & $\begin{array}{l}147-108,5 \\
152,5-104,5\end{array}$ \\
\hline $\begin{array}{l}\text { ASVM4 } \\
\text { ASVM4 }\end{array}$ & $\begin{array}{l}1979 \\
1980\end{array}$ & $\begin{array}{l}24 \\
32\end{array}$ & $\begin{array}{l}119,73 \\
112,96\end{array}$ & $\begin{array}{l}8,53 \\
8,24\end{array}$ & $\begin{array}{l}133,5-97,5 \\
135-99\end{array}$ \\
\hline $\begin{array}{l}\text { AS68 FL } \\
\text { AS68 FL }\end{array}$ & $\begin{array}{l}1979 \\
1980\end{array}$ & $\begin{array}{r}2 \\
11\end{array}$ & $\begin{array}{l}104,25 \\
111,95\end{array}$ & $\begin{array}{l}2,47 \\
8,92\end{array}$ & $\begin{array}{l}106-102,5 \\
131,5-98,5\end{array}$ \\
\hline $\begin{array}{l}\text { AS68 VM4 } \\
\text { AS68 VM4 }\end{array}$ & $\begin{array}{l}1979 \\
1980\end{array}$ & $\begin{array}{r}4 \\
13\end{array}$ & $\begin{array}{l}106,13 \\
105,54\end{array}$ & $\begin{array}{r}7,26 \\
13,26\end{array}$ & $\begin{array}{l}116-99 \\
127,5-92,5\end{array}$ \\
\hline $\begin{array}{l}\text { Moyenne des familles } \\
\text { Moyenne des familles }\end{array}$ & $\begin{array}{l}1979 \\
1980\end{array}$ & $\begin{array}{l}50 \\
85\end{array}$ & $\begin{array}{l}127,41 \\
117,88\end{array}$ & & \\
\hline $\begin{array}{l}\text { Capitole } \\
\text { Capitole }\end{array}$ & $\begin{array}{l}1979 \\
1980\end{array}$ & & $\begin{array}{l}90,00 \\
92,25\end{array}$ & & \\
\hline $\begin{array}{l}\text { Comtal } \\
\text { Comtal }\end{array}$ & $\begin{array}{l}1979 \\
1980\end{array}$ & & $\begin{array}{r}99,50 \\
102,00\end{array}$ & & \\
\hline
\end{tabular}

Nous avons obtenu des lignées à la fois plus courtes et plus résistantes à la septoriose que «Comtal»; le type de matériel obtenu pourrait permettre de rompre plus facilement la liaison entre la résistance et la taille des plantes, la liaison observée dans ce matériel étant plus faible que celle que nous avons trouvée pour des lignées en sélection présentant la même amplitude de variation de taille.

On constate que le matériel qui a été recroisé précocément est, en moyenne, un peu moins résistant que celui qui n'a pas été recroisé mais ce dernier est de taille plus haute et plus éloigné d'un type agronomique acceptable. Du seul point de vue de la résistance à la septoriose, il pourrait donc être plus efficace de sélectionner les lignées les plus résistantes et de différer leur recroisement plutôt que de pratiquer ces recroisements directement sur le matériel en disjonction. Un compromis entre ces 2 techniques est peutêtre à rechercher si l'on veut obtenir rapidement du matériel de valeur agronomique acceptable et amélioré du point de vue de la résistance à la septoriose.

Le matériel issu de l'hybride AS présenté ici constitue une

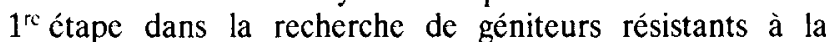
septoriose. C'est pourquoi nous n'avons pas renouvelé le 
croisement afin de vérifier les hypothèses le concernant. Depuis nous avons créć des amphiplö̈des (Ae, squarrosa $\times$ Triticum $4 \times$ ) à $2 \mathrm{n}=42$ chromosomes (TROTTET et al., 1982). L'obtention de tels amphidiplö̈des va permettre de mieux utiliser la variabilité apportée par cet Aegilops. d'analyser les relations entre le génome D d'Ae. squarrosa ct les autres génomes du blé tendre, d'étudier l'hérédité de la résistance apportéc par Ae. squarrosa et d'exploiter de façon optimale ce géniteur.

\section{RÉFÉRENCES BIBLIOGRAPHIQUES}

Bourgeois Françoise, Dosba Françoise, Douaire G., 1978. Analyse et identification des translocations réciproques présentés chez le géniteur «VPM » et les variétés « Marne», «Moisson» et «Roazon». Ann. Amélior. Plant., 28 (4), 411-429.

Brönnimann A., 1974. Bisherige Erfahrungen in der Züchtung auf Septoria-Toleranz (Septoria nodorum Bcrk.) bei Weizen. Rech. agron. Suisse, 15 (3/4), 491-500.

Dosba Françoise, Doussinault G., 1978. Création de lignées présentant les caractéristiques agronomiques favorables d'Aegilops ventricosa. Ann. Amélior. Plant., 28 (1), 27-44.

Fossati A., Brönnimann A., 1975. Obtention de mutants tolérants à
Septoria nodorum Berk. chez le blé tendre (Triticum aestivum L.). Z. Pflanzenzücht., 75, 205-216.

Jahier J., Trottet M., 1980. Consequences of an attack of Septoria nodorum on the accumulation of dry matter in the grain of an accession of Aegilops squarrosa. Cereal Res. Com., 8 (2), 325-330.

Trottet M., Jahier J., Tanguy Anne-Marie, 1982. A study of an amphiploid between Aegilops squarrosa Tausch. and Triticum dicoccum Schubl. Cereal Res. Com., 10 (1-2), 55-59.

Trottet M., Merien P., 1982. Analyse du comportement de vingt lignées de blé tendre vis-à-vis de Septoria nodorum Berk. Agronomie, 2 (8), 727-734 